\title{
A New Unified Scheme for Controlled Power System Separation Using Synchronized Phasor Measurements
}

\author{
Kai Sun, Member, IEEE, Kyeon Hur, Member, IEEE, and Pei Zhang, Senior Member, IEEE
}

\begin{abstract}
Controlled power system separation, which separates the transmission system into islands in a controlled manner, is considered the final resort against a blackout under severe disturbances, e.g., cascading events. Three critical problems of controlled separation are where and when to separate and what to do after separation, which are rarely studied together. They are addressed in this paper by a proposed unified controlled separation scheme based on synchrophasors. The scheme decouples the three problems by partitioning them into sub-problems handled strategically in three time stages: the Offline Analysis stage determines elementary generator groups, optimizes potential separation points in between, and designs post-separation control strategies; the Online Monitoring stage predicts separation boundaries by modal analysis on synchrophasor data; the Real-time Control stage calculates a synchrophasor-based separation risk index for each boundary to predict the time to perform separation. The proposed scheme is demonstrated on a 179-bus power system by case studies.
\end{abstract}

Index Terms-Cascading events, inter-area oscillation, load shedding, modal analysis, out of step, phasor measurement unit, synchrophasor, system islanding, system separation.

\section{INTRODUCTION}

$\mathbf{M}$ ANY efforts have been made to avoid blackouts in North America since the Northeast Blackout of 1965 [1]-[4]. However, cascading events leading to catastrophic blackouts continued to happen on power transmission systems in the North America as well as other countries. Severe disturbances close to interfaces between interconnected control areas, coming with a sequence of local protective relay actions, may lead to collapse of the network into two or more islands. An island formed unintentionally may be unsustainable if its generators are out of step or lack capacities to balance its load, or any of its components are overloaded to cause more relay actions. Thus, network collapse may continue in unsustainable islands to result in large-area power outages. Controlled system

Manuscript received April 19, 2010; revised August 15, 2010 and October 15, 2010; accepted December 03, 2010. Date of publication January 20, 2011; date of current version July 22, 2011. This work was supported by the Electric Power Research Institute (EPRI). Paper no. TPWRS-00307-2010.

K. Sun and P. Zhang are with the Electric Power Research Institute, Palo Alto, CA 94304 USA (e-mail: ksun@epri.com; pzhang@epri.com).

K. Hur is with Yonsei University, Seoul, Korea (e-mail: khur@yonsei.ac.kr). Color versions of one or more of the figures in this paper are available online at http://ieeexplore.ieee.org.

Digital Object Identifier 10.1109/TPWRS.2010.2099672 separation (or islanding) is considered the final resort against a blackout under severe disturbances. Rather than letting transmission network collapse by itself, the control center may separate it in a controlled manner to ensure that, in every island formed, sufficient loads can survive and are securely supported by stabilized local generators through the island's transmission sub-network. The formation of islands if properly designed will also make their resynchronization easier, towards a prompt system restoration. A practical controlled separation scheme needs to address three critical problems:

- Where to separate? (i.e., the separation points to form sustainable islands)

- When to separate? (i.e., separation timing)

- What to do after separation? (i.e., post-separation control actions in formed islands, e.g., generation rejection and load shedding)

In addition, a controlled separation scheme should consider what hardware devices are supposed to execute separation and how to coordinate them at multiple locations.

There are increasing studies on controlled separation, but the three problems are rarely studied together under one framework although they are, in fact, coupled. Most studies focus on solving either "where" or "when" and give some consideration to "what". Papers [5]-[7] utilize graph theory-based techniques to simplify a power network and then adopt heuristic ordered binary decision diagram (OBDD)-based methods to solve the separation points addressing generator coherency, generation-load balance, and transmission capacity constraints in each island. Further simulation and implementation studies on OBDD-based methods are presented in [8] and [9]. Paper [10] integrates OBDD-based methods with single-machine-equivalent techniques [11], [12] to study formation of stable islands. Papers [13]-[16] present slow coherency-based controlled islanding techniques, which identify the weakest network connection to form islands that are readily stabilized. Papers [17] and [18] utilize particle swarm optimization techniques to solve separation points. The above papers focus on addressing problem "where". For problem "when", out-of-step relays are traditionally applied to protect generators or disconnect two control areas once detecting any out-of-step condition from local measurements, but their designs do not adapt to coordination at the system level. Papers [19] and [20] apply heuristic decision tree-based algorithms to determine separation time by means of phasor measurement units (PMUs).

Synchrophasors, e.g., PMUs, are being extensively installed in power transmission systems to help control centers monitor 
wide-area system dynamic behaviors [21]. They can provide accurately synchronized phasor measurements at a high-sampling rate, e.g., 30 samples per second. Their online data can play critical roles in controlled system separation, e.g., monitoring inter-area oscillations to analyze potential separation boundaries, estimating the risk of system separation to predict separation time, detecting frequency and voltage excursions in formed islands, and assisting in re-synchronizing islands to restore the system.

This paper will propose a new unified synchrophasor-based controlled separation scheme addressing the three problems mentioned above. They are partitioned into sub-problems reassembled by time frame and addressed in three time stages. The scheme not only decouples the three problems but also effectively schedules computational resources to enable online implementation. Especially, synchrophasors are employed to answer "where" and "when": a concept of "elementary generator group" is introduced to predetermine potential separation boundaries and place synchrophasors and separation hardware; modal analysis techniques are applied to synchrophasor data to online predict the most probable ones from predetermined separation boundaries; in real time, a separation risk index is calculated at each predicted boundary using synchrophasor data to determine the separation time.

In the rest of the paper, Section II first introduces key ideas and stages of the scheme and then presents the algorithms of each stage in detail. In Section III, the scheme is demonstrated on a 179-bus power system. Finally, some conclusions are drawn in Section IV.

\section{Proposed Controlled Separation Scheme}

\section{A. Overview of the Scheme}

The proposed controlled separation scheme assumes that separation relays (SRs for short) can be installed at predetermined separation points and coordinated at the system level. Once separation is needed, the SRs on selected locations are tripped simultaneously while the others are blocked to form desired islands. The scheme is implemented strategically in three time stages, i.e., Offline Analysis (OFA) stage, Online Monitoring (ONM) stage, and Real-time Control (RTC) stage to addresses the three problems as follows.

- "Where": the OFA stage reduces the search scope for solutions to a set of potential separation boundaries, and on that basis, final separation boundaries are determined adaptively in the ONM stage.

- "When": the RTC stage predicts separation time by realtime synchrohpasor data and modal analysis results from the ONM stage.

- "What": post-separation control strategies (e.g., load shedding and generation rejection) are designed for each potential island in the OFA stage and are finally selected to perform in the RTC stage.

Tasks and objectives of each stage are given in Table I. Detailed tasks and algorithms will be introduced below.

\section{B. Offline Analysis (OFA) Stage}

System planners of a power system may perform the following tasks for typical operating conditions:
TABLE I

THREe STAGES OF THE PRoposed SCHEME

\begin{tabular}{|c|c|c|}
\hline Stages & Main Tasks & $\begin{array}{c}\text { Addressed } \\
\text { Problems } \\
\end{array}$ \\
\hline $\begin{array}{l}\text { Offline } \\
\text { Analysis } \\
\text { /OFA } \\
\text { (in the } \\
\text { planning } \\
\text { stage) } \\
\end{array}$ & $\begin{array}{l}\text { - Identify elementary coherent groups of } \\
\text { generators and potential separation } \\
\text { boundaries forming sustainable islands } \\
\text { - Place SRs and synchrophasors. } \\
\text { - Develop a post-separation control } \\
\text { strategy for each potential island } \\
\end{array}$ & $\begin{array}{l}\text { Study } \\
\text { "where", } \\
\text { "when", } \\
\text { and } \\
\text { "what" }\end{array}$ \\
\hline $\begin{array}{c}\text { Online } \\
\text { Monitoring } \\
\text { /ONM } \\
\text { (periodically) }\end{array}$ & $\begin{array}{l}\text { - Monitor oscillations between } \\
\text { elementary coherent groups } \\
\text { - Predict currently probable separation } \\
\text { boundaries from those pre-determined } \\
\text { in the OFA stage. }\end{array}$ & $\begin{array}{l}\text { Solve } \\
\text { "where" } \\
\text { and study } \\
\text { "when" }\end{array}$ \\
\hline $\begin{array}{c}\text { Real-time } \\
\text { Control/RTC } \\
\text { (in real time) }\end{array}$ & $\begin{array}{l}\text { - Use real-time synchrophasor data to } \\
\text { estimate the separation risk on each } \\
\text { boundary predicted in the ONM stage } \\
\text { - Form islands at an boundary when its } \\
\text { risk exceeds a preset threshold } \\
\text { - Perform post-separation control } \\
\text { strategies from the OFA stage in islands }\end{array}$ & $\begin{array}{l}\text { Solve } \\
\text { "when" } \\
\text { and } \\
\text { "what" }\end{array}$ \\
\hline
\end{tabular}

- analyze generator coherency to place synchrophasors;

- determine potential separation points to place SRs;

- develop post-separation control strategies.

1) Analyze Generator Coherency to Place Synchrophasors: Following disturbances, generators tend to form coherent groups with regard to slow oscillation modes [22]. Under different operating conditions, actual generator grouping may change. For example, one previously coherent group may separate into smaller groups, or by contraries, multiple groups may combine to a bigger coherent group. In order to give consideration to probable changes in generator grouping under credible operating conditions, the proposed scheme separates all generators into a number (say $N_{G}$ ) of elementary coherent groups (ECGs for short), whose combinations in different ways can cover most scenarios of generator grouping. ECGs can be determined by slow-coherency analysis techniques in [14] and [22]. In addition, simulations on a list of credible "N-k" contingencies can help verify or modify those ECGs. For instance, if any contingency breaks the synchronism of one ECG and separation of its generators into different islands is acceptable for the control center, the ECG may further be partitioned into smaller ECGs to give consideration to that scenario. It should be pointed out that the strategy of partitioning ECGs unnecessarily covers too many different scenarios of generator grouping since controlled separation is the last resort against a blackout and is designed for extreme conditions, e.g., cascading events leading to severe out-of-step between control areas.

Another factor that may make the boundaries of coherent groups be ambiguous is the existence of "fuzzy" generators that could be included in different ECGs when the operating condition changes. Generally, such a generator does not have an overwhelmingly stronger coherency with one adjacent coherent group than the others. That, in fact, increases the probability of stabilizing it with a preselected adjacent coherent group if they together form one island. Thus, a "fuzzy" generator can be handled as follows: if it has a big capacity and loss of it after system separation is unexpected, it may be treated as an ECG by itself; otherwise, it can be assigned to an adjacent ECG 


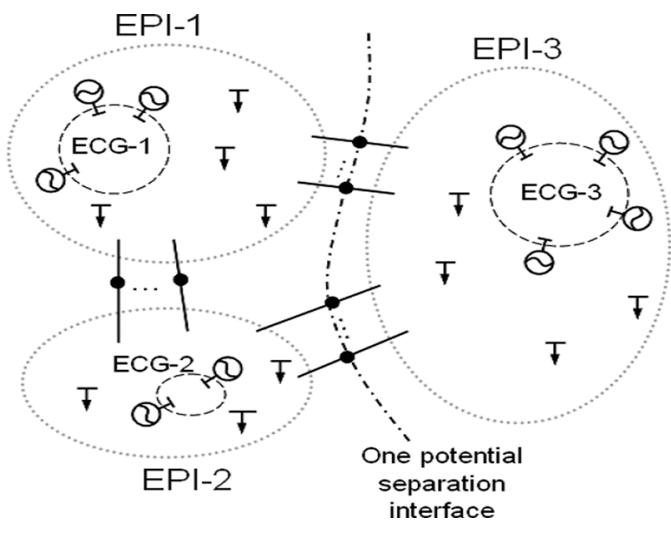

Fig. 1. Power system with three ECGs.

that is either geographically closer or needs more additional generation to balance nearby loads, and it is not synchronous with the ECG after system separation, it could be tripped. In practice, system planners have ideas about potential separation boundaries and the number of islands under both technical and nontechnical concerns. Accordingly, the number and partition of ECGs can be defined. The proposed scheme will deal with out-of-step only between the defined ECGs, and handle instability within an ECG by post-separation control actions, e.g., tripping unstable generators or shedding extra load.

The next step is to place synchrophasors in order to monitor oscillations between ECGs and predict separation boundaries. At least one synchorphasor is needed for an ECG. For more reliable results, it is recommended to place multiple synchrophasors at the terminals of main generators of each ECG to measure their rotor angles, whose mean value represents the average rotor angle of that ECG. More discussion on synchrophasor placement for the purpose of oscillation mode monitoring can be found in [23]. To illustrate ECGs and potential islands, Fig. 1 shows an example with three ECGs, i.e., ECG-1, ECG-2, and ECG-3, as circled by broken lines. Assume that they have, respectively, 3, 2, and 4 big generators, each monitored by a synchrophasor.

2) Determine Potential Separation Points to Place SRs: For typical operating conditions, identify a set of branches to place SRs as potential separation points, which enable isolating each ECG with matched load as one island satisfying the following.

- Generation-load imbalance is minimized for typical operating conditions or less than preset threshold $\Delta P$.

- No transmission capacity limit is violated.

The selection of $\Delta P$ is discussed in [5]. Fast separation-point searching algorithms, e.g., OBDD-based methods in [5] and [7], can be applied here. The $N_{G}$ potential islands that, respectively, contain $N_{G}$ ECGs are named elementary potential islands (EPIs for short), denoted by EPI $-1 \sim \mathrm{EPI}-N_{G}$. Fig. 1 circled three EPIs by dotted lines and place SRs on the branches between them as potential separation points shown by black dots. In fact, any EPIs directly connected may merge to one island if their ECGs keep synchronous. In Fig. 1, EPI-1+EPI-2, EPI-1+EPI-3, and EPI-2+EPI-3 are three potential merged islands. Thus, the system has six potential islands and three potential boundaries. For a generic case, the total number of potential islands (EPIs or their combinations) reaches its maximum value $2^{N_{G}}-2$ when all EPIs are directly connected, i.e., aggregating nodes in each EPI forms an $N_{G}$-node complete graph. SRs need to be placed at all potential boundaries. When separation is needed, only the SRs between out-of-step ECGs are tripped and the others are blocked. As an example, Fig. 1 draws one potential separation boundary involving four SRs to form two islands: EPI-1+ EPI-2 versus EPI-3.

3) Develop Post-Separation Control Strategies: Once an island is formed, it may require post-separation control to arrest frequency excursions and quickly stabilize generators, e.g., shedding extra load or reject extra generation. For every potential island, the amounts and locations of control can be studied offline and give considerations to different operating conditions. Thus, a strategy table with a list of post-separation control strategies corresponding to different operating conditions can be developed for each potential island as follows: 1) define a number of representative typical power-flow profiles, e.g., operating conditions of peak load, light load, and several load levels in between with proper generation dispatches; 2) for each power-flow profile, perform simulations to determine the amount of reduced load or generation in each potential island. After the island is formed, perform the strategy that matches best with the current operating condition.

\section{Online Monitoring (ONM) Stage}

Angle separation between control areas generally happens on dominant inter-area modes. In this stage, at every time step of $\Delta T$, synchrophasor data over the last time window of $T$ are monitored to

- identify dominant inter-area modes;

- predict probable separation boundaries.

$T$ is unnecessarily too long. Since inter-area oscillation frequencies are mainly in $0.1 \sim 0.7 \mathrm{~Hz}$ [24], $T>20 \mathrm{~s}$ would enable identification of the slowest frequency. Generally, $T=20 \sim$ $60 \mathrm{~s}$ is recommended. $\Delta T$ equals one to several seconds longer than the computational time in this stage.

1) Identify Dominant Inter-Area Modes: At every time step of $\Delta T$, continuous synchrophasor data about $\delta_{1} \sim \delta_{N_{G}}$ over the past $T$ are obtained. Calculate angle difference $\delta_{i j}=\delta_{i}-\delta_{j}$ for any two adjacent ECGs. Apply fast Fourier transform (FFT) to each $\delta_{i j}$ to identify the frequencies of several (e.g., two or three) strongest oscillation modes in terms of their magnitudes. Thus, a number of modes are identified as candidates for the system. Represent the modes with very close frequencies by one mode with their average frequency and magnitude. Finally, a few representative modes with distinct frequencies are identified.

Apply the modal analysis technique in [23] to judge whether a representative mode is an inter-area or local mode. Introduce squared-coherency function $\gamma_{i j}^{2}$ as defined in (1) about $\delta_{i}$ and $\delta_{j}$, where $\omega$ is the angular frequency of that representative mode, $S_{i j}(\omega)$ is the cross-spectral density (CSD) of $\delta_{i}$ and $\delta_{j}, S_{i i}(\omega)$ and $S_{j j}(\omega)$ are, respectively, their power spectral densities (PSD), and $\boldsymbol{F}(\cdot)$ denotes FFT:

$$
\begin{aligned}
\gamma_{i j}^{2}(\omega) & =\frac{\left|S_{i j}(\omega)\right|^{2}}{S_{i i}(\omega) S_{j j}(\omega)} \\
& =\frac{E\left\{F^{*}\left(\delta_{i}\right) \cdot F\left(\delta_{j}\right)\right\}}{E\left\{F^{*}\left(\delta_{i}\right) \cdot F\left(\delta_{i}\right)\right\} \cdot E\left\{F^{*}\left(\delta_{j}\right) \cdot F\left(\delta_{j}\right)\right\}} .
\end{aligned}
$$


CSD and PSD can be estimated using the data of $\delta_{i}$ and $\delta_{j}$ over the past $T$. If $\gamma_{i j}^{2}(\omega)$ is close to 1 (e.g., $>0.7$ ) for some $i$ and $j$, the mode is considered an inter-area mode rather than a local mode; otherwise, it is filtered out. Order the identified inter-area modes by magnitude, and select the top- $N_{M}$ (e.g., $2 \sim 5$ ) as dominant inter-area modes with $\omega_{1} \sim \omega_{N_{M}}$ (frequencies $f_{1} \sim$ $\left.f_{N_{M}}\right)$.

2) Predict Probable Separation Boundaries: For any ECG- $i$ and ECG- $j$, the phase angle of $S_{i j}$ is denoted by $\beta_{i j}$ and can be calculated to estimate the mode shape at any $\omega$. If $\left|\beta_{i j}(\omega)\right| \approx$ $0^{\circ}$, two ECGs swing together with $\omega$; if $\left|\beta_{i j}(\omega)\right| \approx 180^{\circ}$, they swing against each other. In implementation, we may define two thresholds $\beta_{1}<90^{\circ}$ and $90^{\circ}<\beta_{2}<180^{\circ}$ of $\beta_{i j}$ (e.g., $\beta_{1}=$ $60^{\circ}$ and $\beta_{2}=120^{\circ}$ ) and adopt the following algorithm to infer potential separation boundaries.

1) Calculate $\beta_{i j}\left(\omega_{n}\right)$ for each dominant inter-area mode $\omega_{n}$ $\left(n=1 \sim N_{M}\right)$. If $\left|\beta_{i j}\left(\omega_{n}\right)\right|<\beta_{1}$, ECG- $i$ and ECG- $j$ are treated as "swinging together with $\omega_{n}$ "; if $\left|\beta_{i j}\left(\omega_{n}\right)\right|>\beta_{2}$, they are treated as "swinging against each other with $\omega_{n}$ "; otherwise, the relationship is "undetermined with $\omega_{n}$ ".

2) If a cut set can be determined from the above analysis, it indicates a probable separation boundary with $\omega_{n}$; otherwise, no boundary is associated with $\omega_{n}$.

3) Order all determined boundaries by the magnitudes of the associated modes, and select the top $N_{I}$ as probable separation boundaries to monitor in the RTC stage.

Each probable separation boundary $k\left(k=1 \sim N_{I}\right)$ divides $\delta_{1} \sim \delta_{N_{G}}$ into two groups. Calculate the averages of both groups in the past $T$, and denote their differences by $\Delta_{k}$. Then, estimate damping ratio $\zeta_{k, n}$ of mode $\omega_{n}$ in $\Delta_{k}$ by Prony analysis [25] or analytical wavelet transform (AWT) [26].

It should be noted that the ONM stage only observes synchrophasor measurements to predict separation boundaries, without making any assumption about disturbances. If disturbances happen between two ECGs and impact their interface, that interface will have a high probability to be part of the final separation boundary. If the disturbances are detectable in real time in the system, the predicted separation boundaries may be revised to contain that interface; if not, because the impacts from disturbances can still be captured in real time by synchrophasors, the calculation results of the ONM and RTC stages can generally reflect those impacts by, e.g., stronger modes relevant to that interface or increased separation risks on the boundaries containing the interface.

\section{Real-Time Control (RTC) Stage}

Based on the latest results from the ONM stage, at every time step of $\Delta t(\ll \Delta T$, e.g., $0.1 \mathrm{~s})$ :

- estimate angle separation risks on predicted boundaries;

- form islands and take post-separation control actions.

1) Estimate Angle Separation Risks on Predicted Boundaries: At a time step, assume $t=\tau$ and consider boundary $k$ $\left(k=1 \sim N_{I}\right) . \Delta_{k}(t)$ during $[\tau, \tau+\Delta t]$ can be approximated by summing damped sinusoidal signals $\alpha_{k, 1}(t) \sim \alpha_{k, N_{M}}(t)$ about $N_{M}$ dominant inter-area modes and a steady-state signal $A_{k, 0}(\tau)$ :

$$
\begin{aligned}
\Delta_{k}(t) \approx & A_{k, 0}(\tau)+\sum_{n=1}^{N_{M}} \alpha_{k, n}(t) \\
= & A_{k, 0}(\tau)+\sum_{n=1}^{N_{M}} A_{k, n}(\tau) e^{\frac{-\zeta_{k, n} \omega_{n}(t-\tau)}{\sqrt{1-\zeta_{k, n}^{2}}}} \\
& \times \cos \left(\omega_{n} t+\phi_{k, n}\right) .
\end{aligned}
$$

$A_{k, 0}(\tau)$ can be estimated by averaging $\Delta_{k}(t)$ over time window $[\tau-T, \tau]$. Amplitudes $A_{k, 1}(\tau) \sim A_{k, N_{M}}(\tau)$ can be solved from (2) together with equations in (3), where $\Delta_{k}(t)$ 's derivatives of different orders are estimated using its measurements near $t=\tau$ :

$$
\left\{\begin{array}{l}
\dot{\Delta}_{k}(\tau)=\dot{\alpha}_{k, 1}(\tau)+\ldots+\dot{\alpha}_{k, N_{M}}(\tau) \\
\ldots \\
\Delta_{k}^{\left(2 N_{M}-1\right)}(\tau)=\alpha_{k, 1}^{\left(2 N_{M}-1\right)}(\tau)+\ldots+\alpha_{k, N_{M}}^{\left(2 N_{M}-1\right)}(\tau)
\end{array} .\right.
$$

Define

$$
A_{k}(\tau)=A_{k, 0}(\tau)+A_{k, 1}(\tau)+\ldots+A_{k, N_{M}}(\tau)
$$

which is an estimate of the theoretically maximum value that $\Delta_{k}$ might reach in an immediate period $T^{\prime}=1 / \min \left(f_{1}, \ldots, f_{N_{M}}\right)$ and is important in estimating the risk of angle separation. If $\alpha_{k, 1}(t) \sim \alpha_{k, N_{M}}(t)$ are extracted from $\Delta_{k}(t)$, e.g., by bandpass filters, $A_{k}(\tau)$ may also be calculated directly by

$$
\begin{aligned}
A_{k}(\tau) & =A_{k, 0}(\tau) \\
& +\sum_{n=1}^{N_{M}} \sqrt{\alpha_{k, n}^{2}(\tau)+\left(\frac{\dot{\alpha}_{k, n}(\tau)}{\omega_{n}}+\frac{\zeta_{k, n} \alpha_{k, n}(\tau)}{\sqrt{1-\zeta_{k, n}^{2}}}\right)^{2}}
\end{aligned}
$$

For the simplest case with $N_{M}=N_{I}=1$, only the first interarea mode is used to predict angle separation at one probable separation boundary. Ignore subscript $k$ in (5). There is

$$
A(\tau)=A_{0}(\tau)+\sqrt{\alpha^{2}(\tau)+\left(\frac{\dot{\alpha}(\tau)}{\omega}+\frac{\zeta \alpha(\tau)}{\sqrt{1-\zeta^{2}}}\right)^{2}}
$$

where $\alpha(t)=\Delta(t)-A_{0}(\tau)$.

Regard the two potential islands divided by boundary $k$ as two equivalent generators. Then, an unstable equilibrium point of $\Delta_{k}(t)$ is estimated at $180^{\circ}-A_{k, 0}$, which is the upper limit of $\Delta_{k}(t)$ without causing angle separation. Define the following separation risk index as a real-time indicator of the risk of angle separation on boundary $k$ :

$$
S R I_{k}(\tau)=A_{k}(\tau) \times 100 \% /\left[180^{\circ}-A_{k, 0}(\tau)\right]
$$

In addition, an approximate approach to fast computation of $A_{k}(\tau)$ is to apply FFT to $\Delta_{k}(t)$ over $[\tau-T, \tau]$, denote the FFT magnitudes at $\omega_{1} \sim \omega_{N_{M}}$ by $\hat{A}_{k, 1}(\tau) \sim \hat{A}_{k, N_{M}}(\tau)$ to 
approximate $A_{k, 1}(\tau) \sim A_{k, N_{M}}(\tau)$, and calculate (8) and then $A_{k}(\tau)$ by (5):

$$
\alpha_{k, n}(t) \approx\left[\Delta_{k}(t)-A_{k, 0}(\tau)\right] \cdot \hat{A}_{k, n}(\tau) / \sum_{n=1}^{N_{M}} \hat{A}_{k, n}(\tau) .
$$

2) Form Islands and Take Post-Separation Control Actions: To decide when to perform controlled separation, $S R I_{k}(k=$ $1 \sim N_{I}$ ) can be compared with a predefined threshold (e.g., $90 \%$ ). Once any $S R I_{k}$ exceeds the threshold, controlled separation should be performed on boundary $k$ immediately to form two islands: trip only the SRs on boundary $k$ while blocking the others; perform the post-separation control strategy associated with each island to stabilize generators and arrest frequency declines. After islands are formed, they will be operated respectively as sub-systems until resynchronized to restore the system. If new severe disturbances happen on an island, the algorithms in the ONM and RTC stages might further separate it into smaller islands when necessary.

It should be noted that at what threshold the decision of controlled separation should be made is not a simple problem. The threshold needs to be customized for a specific power system based on offline studies. The proposed separation risk index provides real-time indication of how urgently controlled separation or alternative nonseparation control should be performed. In real time, that risk index reflects the stress on a probable separation boundary as well as the effect of a control. When $S R I_{k}$ approaches $100 \%$, it indicates a high probability of system separation on boundary $k$ if no control is added timely, but unnecessarily means that controlled separation is the only solution. In fact, other emergency control without separating the system, e.g., reconfiguring load and generation in potential islands may also relieve stress on the boundary to increase stability and lower $S R I_{k}$. However, if offline studies or operational experiences show that controlled separation at a certain level of $S R I_{k}$ may lead to overall less loss than any nonseparation control, a threshold could be set there to enable controlled separation. In addition, setting the threshold also depends on some nontechnical factors, e.g., to what extent separating a power system is acceptable.

\section{CASE STUdies}

In this section, the proposed scheme is demonstrated on a 179-bus system as shown in Fig. 2, which is a simplified WECC ac transmission system with 29 generators, 179 buses, and 263 branches in five zones: Zone 1-A, Zone 1-B, Zone 1-C, Zone 2-A, and Zone 2-B. The power-flow base case used has totally $60.8 \mathrm{GW}$ of load.

\section{A. OFA Stage}

EPRI's dynamic reduction program DYNRED is used to analyze slow coherency of the generators. Small contingencies around the interfaces between five zones are simulated to verify generator grouping patterns under operating conditions around the power-flow base case. As shown in Table II, it is found that generators generally form four coherent groups, which are regarded as ECGs and numbered 0-3. Place synchrophasors in

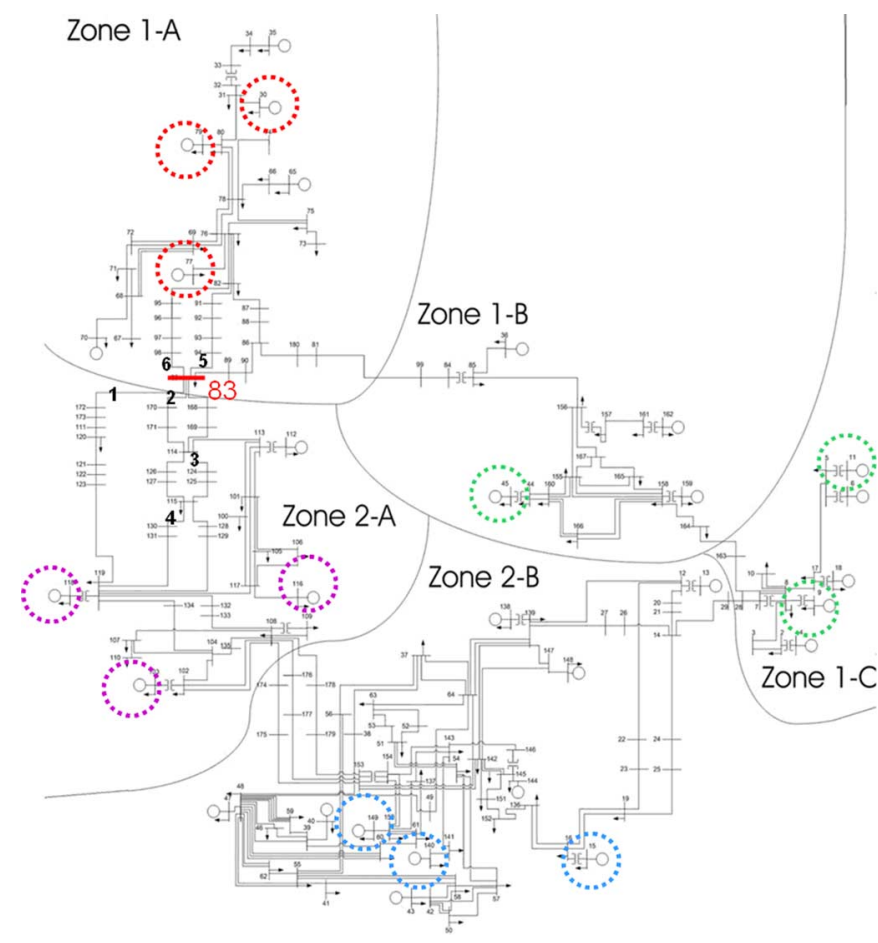

Fig. 2. A 179-bus power system.

TABLE II

ECGS AND SYNCHROPHASOR PLACEMENT

\begin{tabular}{|c|l|c|}
\hline ECGs & \multicolumn{1}{|c|}{ Generator Bus Numbers } & Zone (s) \\
\hline 0 & $\underline{30}, 35,65,70, \underline{77}, \underline{79}$ & $1-\mathrm{A}$ \\
\hline 1 & $\underline{103}, 112, \underline{116}, \underline{118}$ & $2-\mathrm{A}$ \\
\hline 2 & $13, \underline{15}, 40,43,47,138, \underline{140}, 144,148, \underline{149}$ & $2-\mathrm{B}$ \\
\hline 3 & $4,6, \underline{9}, \underline{11}, 18,36, \underline{45}, 159,162$ & $1-\mathrm{B}$ and 1-C \\
\hline
\end{tabular}

each ECG at three largest generators (underlined in Table II and circled in Fig. 2). Totally, 12 generators are monitored, which undertake $62.5 \%$ of the total system load.

According to the method in [5], solve potential separation points as follows.

1) Represent the system by a graph as shown in Fig. 3, where generator and load nodes are, respectively, presented by circles and black dots and four shade areas indicate four ECGs. The load nodes not in shade areas do not contribute to connecting generators of any ECG.

2) Merge each area into an equivalent generator node as indicated by Fig. 3 to simplify the original graph to a 45 -node graph with four equivalent generators numbered $0 \sim 3$. The net power output of each equivalent generator equals the total generation minus load in that area.

3) Apply the OBDD-based algorithm in [5] to quickly find the separation strategies that form four EPIs with generationload imbalance $<2 \mathrm{GW}$ ( $3.3 \%$ of the system load). The following strategy has the minimum imbalances of EPIs and needs the fewest separation points:

a) Interface $0-1$ : lines $83-168,83-170$, and $83-172$;

b) Interface 0-3: line 81-99;

c) Interface 1-2: lines $142-153$ and 153-154;

d) Interface 2-3: line 28-29. 


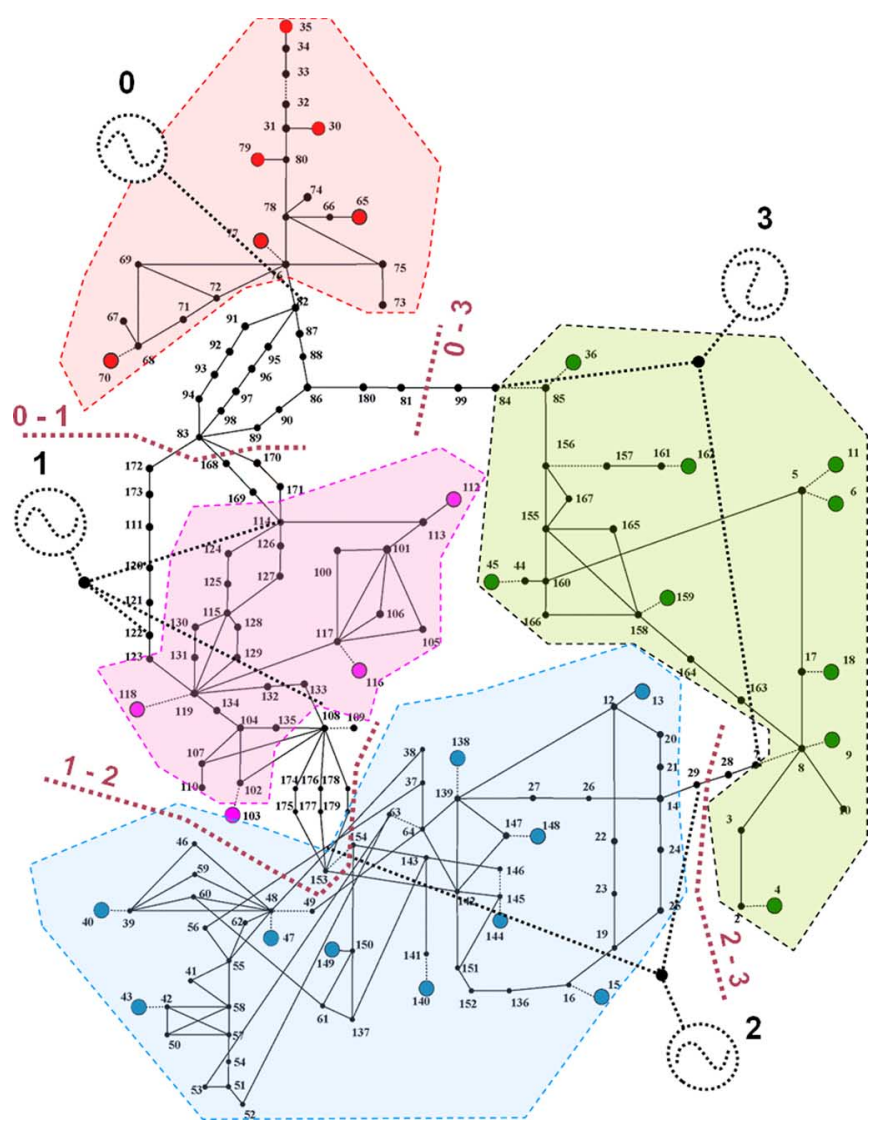

Fig. 3. Graph representing the 179-bus system and its simplified graph.

TABLE III

ELEMENTARY POTENTIAL ISLANDS

\begin{tabular}{|c|c|c|c|}
\hline EPIs & Generation $(\mathrm{GW})$ & Load $(\mathrm{GW})$ & Imbalance $(\mathrm{GW})$ \\
\hline 0 & 28.3 & 26.4 & 1.9 \\
\hline 1 & 5.1 & 6.4 & -1.3 \\
\hline 2 & 15.5 & 17.4 & -1.9 \\
\hline 3 & 12.5 & 10.6 & 1.9 \\
\hline
\end{tabular}

4) Place SRs on the above seven lines.

Those interfaces are shown in Fig. 3. The imbalances of four EPIs are given in Table III.

Time-domain simulations are performed on isolating each potential island and immediately shedding different amounts of load or generation to determine an appropriate amount of control to stabilize the island with frequency in $59.5-60.5 \mathrm{~Hz}$. All 12 potential islands that might be formed and the post-separation control strategies determined for the base case are given in Table IV, where the basis of percentages is the total system load $60.8 \mathrm{GW}$. According to the table, 6 of 12 potential islands can automatically meet the frequency criteria for post-separation operating conditions. The worst case is island No. 7, which sheds $7.3 \%$ load after separation. Similarly, for other typical operating conditions different from the base case, post-separation control strategies can be studied. Finally, a strategy table can be built to cover a range of operating conditions for each potential island.
TABLE IV

CONTROL STRATEgies After SEPARATion

\begin{tabular}{|c|l|l|}
\hline No.'s & Potential Islands & Control Strategies \\
\hline 1 & EPI-0 & Not needed \\
\hline 2 & EPI-1 & Shed 2.2GW (3.6\%) load \\
\hline 3 & EPI-2 & Shed 2.3GW (3.8\%) load \\
\hline 4 & EPI-3 & Not needed \\
\hline 5 & EPI-0 + EPI-1 & Not needed \\
\hline 6 & EPI-0 + EPI-3 & Not needed \\
\hline 7 & EPI-1 + EPI-2 & Shed 4.5GW (7.3\%) load \\
\hline 8 & EPI-2 + EPI-3 & Shed 0.9G (1.5\%) load \\
\hline 9 & EPI-0 + EPI-1 + EPI-3 & Not needed \\
\hline 10 & EPI-0 + EPI-1 + EPI-2 & Shed 2.5GW (4.1\%) load \\
\hline 11 & EPI-1 + EPI-2 + EPI-3 & Shed 3.0GW (4.9\%) load \\
\hline 12 & EPI-0 + EPI-2 + EPI-3 & Not needed \\
\hline
\end{tabular}

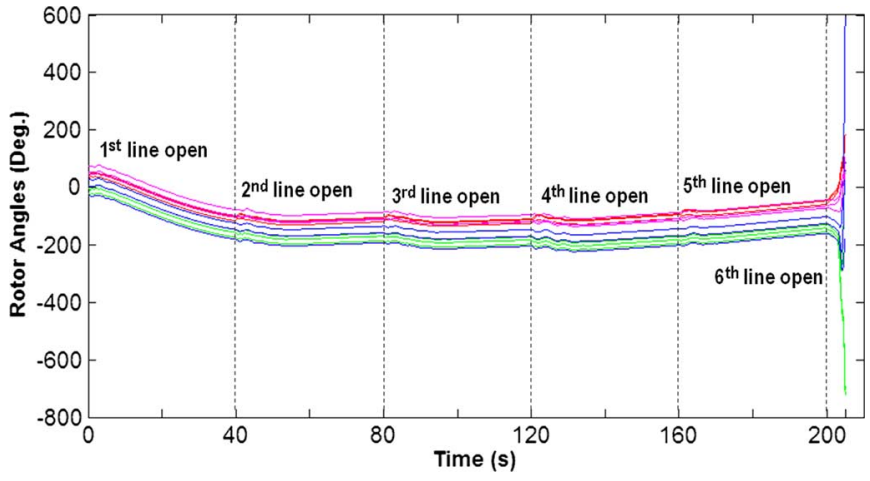

Fig. 4. Rotor angles monitored by 12 synchrophasors.

To demonstrate the ONM and RTC stages of the proposed scheme, six successive three-phase faults are added, respectively, at 0 s, $40 \mathrm{~s}, 80 \mathrm{~s}, 120 \mathrm{~s}, 160 \mathrm{~s}$, and $200 \mathrm{~s}$ on lines $83 \#-172$, 83\#-170, 114\#-124, 115\#-130, 83\#-94, and 83\#-98 (“\#” indicates the fault bus). Fault locations are indicated by numbers 1 $\sim 6$ in Fig. 2. Each fault is cleared after six cycles by tripping the fault line. Those contingencies are close to the interface at bus 83 in the 179-bus system corresponds to the famous California-Oregon Intertie of the WECC system, which was involved in the U.S. western blackout event in August 1996. The contingencies do not break the network connection at that interface but gradually increase the system's vulnerability and lead to instability. Fig. 4 gives rotor angles of the 12 generators monitored by synchrophasors. Instability happens soon after the 6th contingency if no control is added.

\section{B. ONM Stage}

From simulation results, angle differences between four directly connected ECGs are shown in Fig. 5. Those data are assumed to be synchrophasor measurements used in the ONM and RTC stages. From Fig. 5, the steady-state values of $\delta_{01}$ and $\delta_{23}$ are larger than the other two. However, the most probable separation boundary should be predicted based on modal analysis. In MATLAB environment on a desktop PC, the algorithms of the ONM stage take less than $1 \mathrm{~s}$ to finish modal analysis on $60 \mathrm{~s}$ data (30 samples/s) of four angle differences. Let time step 


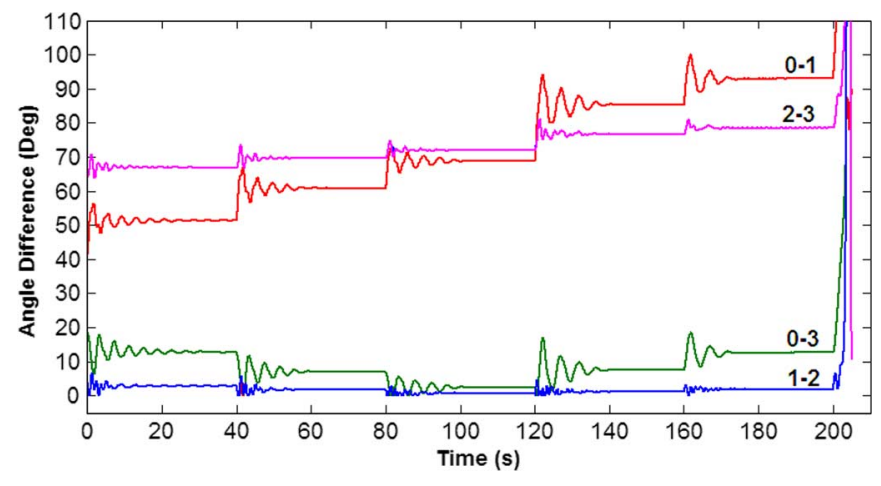

Fig. 5. Angle differences $\delta_{01}, \delta_{03}, \delta_{12}$, and $\delta_{23}$.

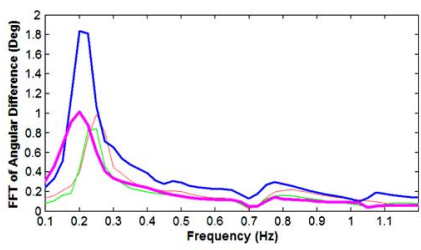

(a)

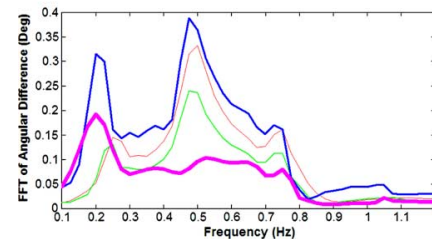

(c)

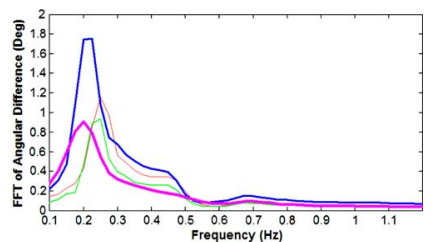

(b)

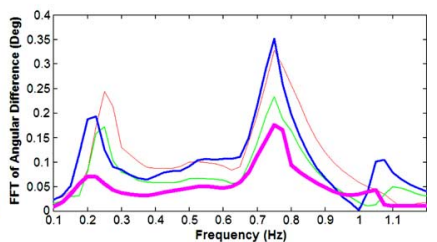

(d)
Fig. 6. FFT on 40-80 s, 80-120 s, 120-160 s, and 160-200 s time windows. (a) $\delta_{01}$. (b) $\delta_{03}$. (c) $\delta_{23}$. (d) $\delta_{12}$.

$\Delta T=1 \mathrm{~s}$ and time window $T=40 \mathrm{~s}$. Thus, the algorithms of the ONM stage are performed every $1 \mathrm{~s}$ on the past $40 \mathrm{~s}$ of data.

To show how inter-area oscillation modes are changed by contingencies, Fig. 6(a)-(d) compares the FFT results of four angle differences on four 40-s time windows, respectively, following contingencies $2,3,4$, and 5 . The results for four time windows are differentiated by increasing line thickness. From the figures, three representative inter-area modes are at $0.25 \mathrm{~Hz}$, $0.5 \mathrm{~Hz}$, and $0.75 \mathrm{~Hz}$. Use the strongest two (i.e., $N_{M}$ ) modes, $0.25 \mathrm{~Hz}$ and $0.75 \mathrm{~Hz}$, to infer separation boundaries. Note that the FFT results on time window 160-200 s are actually seen at $t=200 \mathrm{~s}$ right before the final contingency. At $t=200 \mathrm{~s}$, $\gamma_{i j}^{2}$ and $\beta_{i j}$ for frequencies of $0.1-0.9 \mathrm{~Hz}$ are calculated over $160-200 \mathrm{~s}$ as shown in Fig. 7. Let $\beta_{1}=60^{\circ}$ and $\beta_{2}=120$. From Fig. 7, it can be determined that ECG-0 swings against ECG-1 and ECG-3 at $0.25 \mathrm{~Hz}$, and ECG-1, ECG-2, and ECG-3 swing together at $0.25 \mathrm{~Hz}$. Thus, one (i.e., $N_{I}$ ) probable separation boundary is derived at $t=200 \mathrm{~s}$, which separates EPI-0 (the north island) from the rest of the system (the south island). Thus, strategy 11 in Table IV should be performed to shed $3.0 \mathrm{GW}$ of load in the south island once the four SRs on that boundary are tripped. To study whether the separation boundary is sensitive to $T$, FFT and mode shape analysis are performed on time windows of $35 \mathrm{~s}, 40 \mathrm{~s}$, and $45 \mathrm{~s}$ ending at $t=200 \mathrm{~s}$. They all indicate the same separation boundary. As an example, Fig. 8 shows the results about $\delta_{01}$.

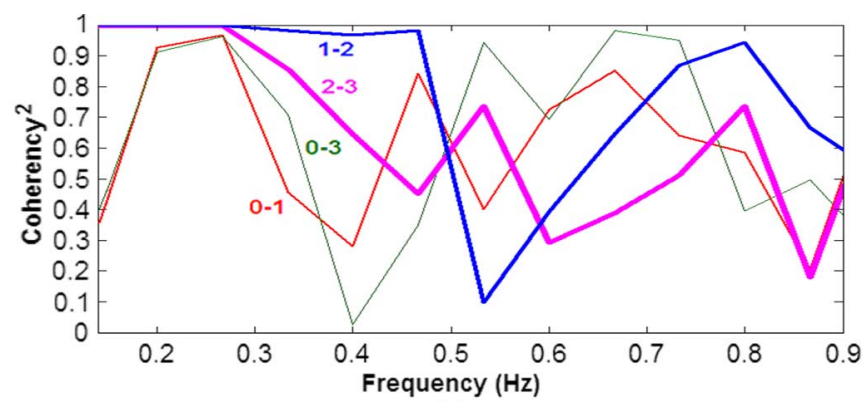

(a)

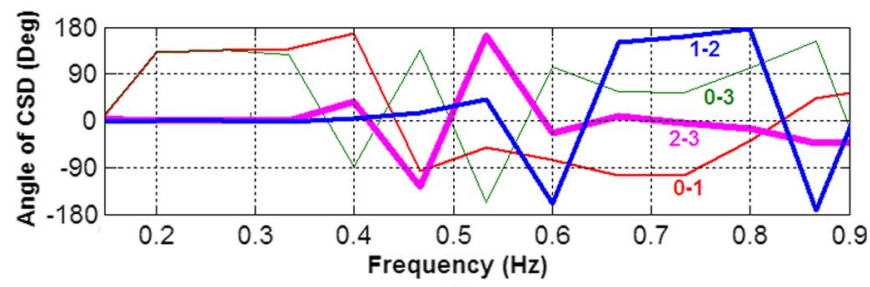

(b)

Fig. 7. Mode shape analysis results at $t=200 \mathrm{~s}$. (a) $\gamma_{i j}^{2}$. (b) $\beta_{i j}$.

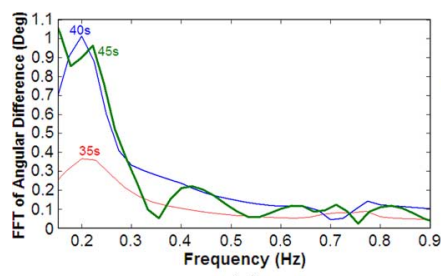

(a)

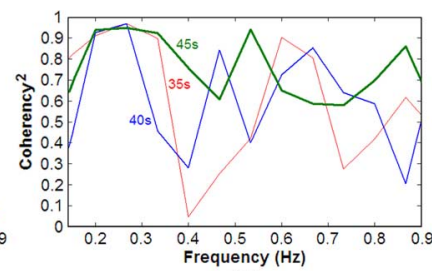

(b)

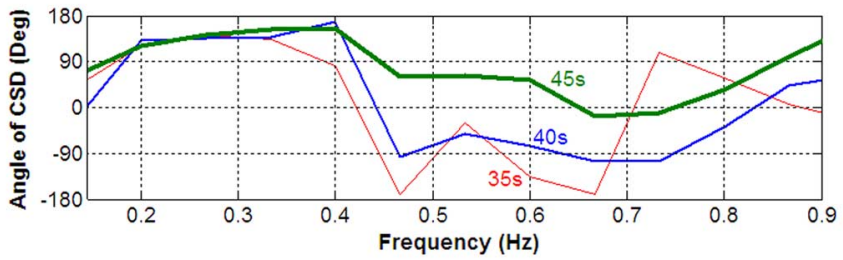

(c)

Fig. 8. Modal analysis on $\delta_{01}$ over $165-200 \mathrm{~s}, 160-200 \mathrm{~s}$, and 155-200 s. (a) FFT results of $\delta_{01}$. (b) $\gamma_{01}^{2}$. (c) $\beta_{01}$.

For that boundary, calculate the angle difference $\Delta(t)$ (ignore subscript 1), i.e., the difference between the average angles of ECG-0 and ECG-1+ECG-2+ECG-3. Fig. 9 shows the calculated $\Delta(t)$, its steady-state value $A_{0}(t)$, and upper limit $180^{\circ}-A_{0}(t)$ for $t=40 \sim 205 \mathrm{~s}$. Damping ratios of the modes at $0.25 \mathrm{~Hz}$ and $0.75 \mathrm{~Hz}$ are estimated by the AWT-based approach every 1 $\mathrm{s}$ for the latest $40-\mathrm{s}$ time window. The results are around $27 \%$ and $6 \%$.

\section{Real-Time Control Stage}

This stage calculates $S R I$ every $\Delta t=0.1 \mathrm{~s}$ on that boundary to predict the time of angle separation. Two approaches are tested: the first approach adopts a low-pass filter and a high-pass filter to extract signals about $0.25 \mathrm{~Hz}$ and $0.75 \mathrm{~Hz}$ from $\Delta(t)$, and then use (5); the second approach adopts (8) and (5) for approximate calculation. Fig. 10 compares the results from two approaches for $t=40 \sim 205 \mathrm{~s}$. The results are very close to each other, and the approximate approach provides slightly lower results during transient periods. From Fig. 10(a), SRI exceeds 


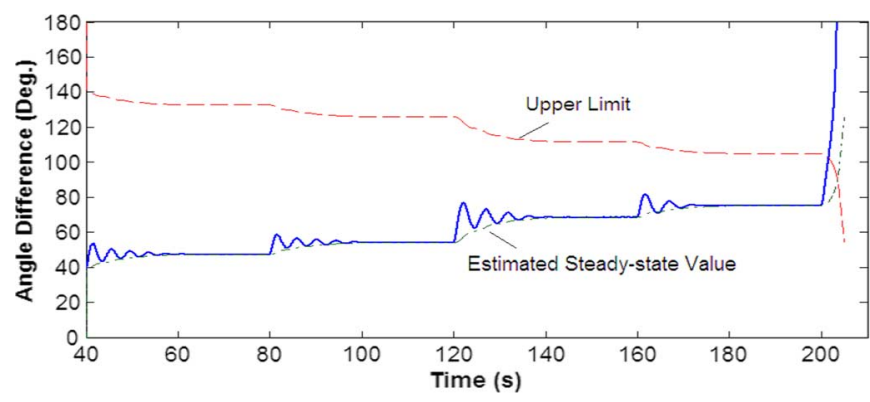

Fig. 9. Angle difference between two potential islands.

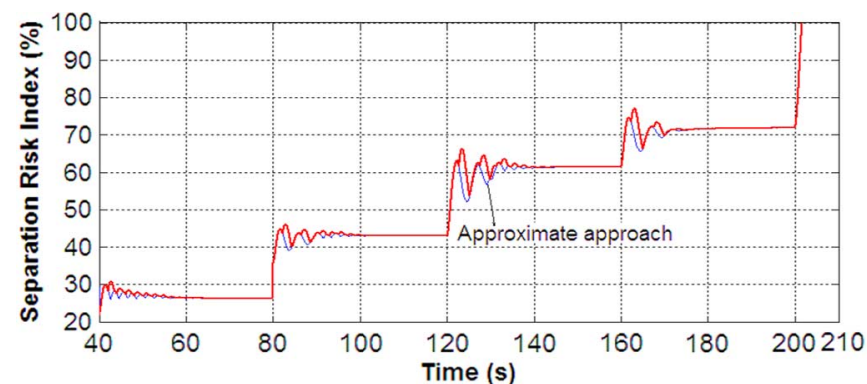

(a)

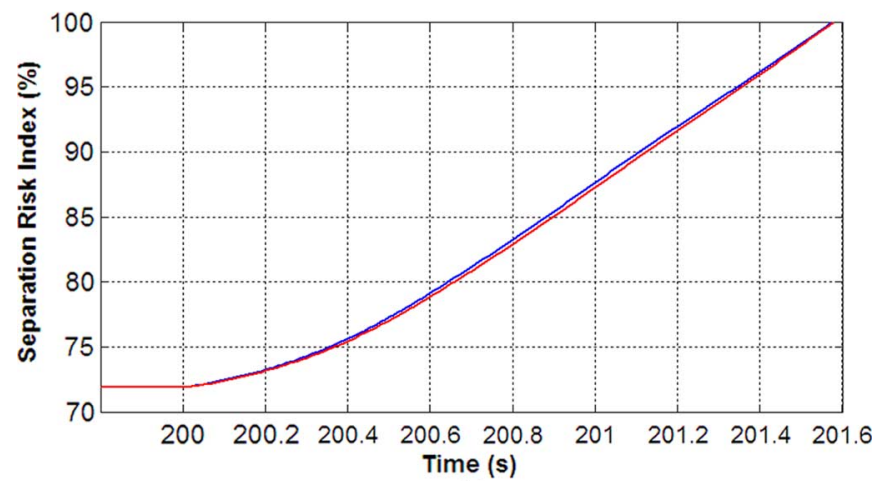

(b)

Fig. 10. Separation risk index.

$60 \%$ after the 4 th contingency (at $t=120 \mathrm{~s}$ ) and then $70 \%$ after the 5 th contingency (at $t=160 \mathrm{~s}$ ). Fig. 10(b) zooms in the period $199.8 \sim 201.6$ s. Following the final contingency, $S R I$ exceeds $90 \%$ at $t=201.1 \mathrm{~s}$ and then $100 \%$ at $t=201.6 \mathrm{~s}$.

Controlled system separation should trip SRs at interfaces $0-1$ and $0-3$ and shed $3 \mathrm{GW}$ in the south island at the same time. That is performed at different times from $t=200 \mathrm{~s}$ to 203 $s$ to test whether the result of separation is sensitive to separation time. Table $\mathrm{V}$ provides the simulation results, showing that if separation is performed before $t=202.5 \mathrm{~s}$, two stable islands can be formed without any other control action. Fig. 11 shows generator frequencies for separation at $t=202.5 \mathrm{~s}$. If controlled separation is performed at $t=202.6 \sim 202.8 \mathrm{~s}$, the north island is still stable spontaneously but generator 112 in the south island loses stability soon after. If it can be tripped by its out-of-step protection, the other generators of the south island will keep stability. If separation is further delayed to $t=203.0 \mathrm{~s}$, four generators in the south island go out of step and need to be tripped. Note that all the above separation scenarios only lose 4.9\% (3.0 $\mathrm{GW}$ ) of the total system load.
TABLE V

COMPARISON OF DIFFERENT SEPARATION TIMES

\begin{tabular}{|c|l|c|}
\hline $\begin{array}{c}\text { Separation } \\
\text { Time (s) }\end{array}$ & \multicolumn{1}{|c|}{ Associated Control Actions } & $\begin{array}{c}\text { Frequencies } \\
\text { (Hz) }\end{array}$ \\
\hline $200.0-202.5$ & Shed 3GW load in the south island & $60.01 \sim 60.14$ \\
\hline $202.6-202.8$ & $\begin{array}{l}\text { Shed 3GW load in the south island and } \\
\text { trip generator 112 }\end{array}$ & $60.01 \sim 60.14$ \\
\hline 202.9 & $\begin{array}{l}\text { Shed 3GW load in the south island and } \\
\text { trip generators 112, 116 and 118 }\end{array}$ & $60.11 \sim 60.14$ \\
\hline 203.0 & $\begin{array}{l}\text { Shed 3GW load in the south island and } \\
\text { trip generators 112,116,118 and 4 }\end{array}$ & $60.11 \sim 60.14$ \\
\hline
\end{tabular}

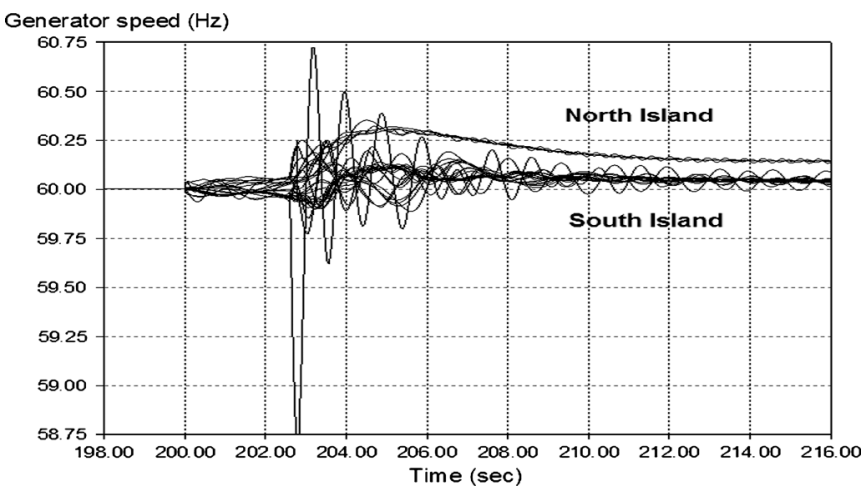

Fig. 11. Generator frequencies after separation at $202.5 \mathrm{~s}$.

\section{Simulations on a Stressed Operating Condition}

The OFA stage is based on a number of representative operating conditions. However, the actual operating condition may change with time to cause a difference from even the closest representative operating condition. Here, an operating condition slightly different from the base case is considered to study the influences on the results from the ONM and RTC stages. Uniformly increase the loads of the base case by $2 \%$ and adjust the generation of Zone 1-A to balance the load increase. In fact, that stresses interface $0-1$. The same sequence of contingencies is added around that interface and, as shown in Figs. 12 and 13, the system loses stability following the 3rd contingency at $80 \mathrm{~s}$ if no control is added. Algorithms of the ONM and RTC stages are performed on angle difference data. Fig. 14 provides 40-s modal analysis results at $t=80 \mathrm{~s}$ (right before the $3 \mathrm{rd}$ contingency). The strongest two inter-area modes are around $0.25 \mathrm{~Hz}$ and $0.75 \mathrm{~Hz}$ and, respectively, indicate two probable separation boundaries:

- Boundary 1 ( $0.25 \mathrm{~Hz}$ mode): interfaces $0-1$ and $0-3$, between EPI-0 and the other EPIs;

- Boundary 2 ( $0.75 \mathrm{~Hz}$ mode): interfaces $0-3$ and $1-2$, between EPI-0+EPI-1 and EPI-2+EPI-3.

At $t=80 \mathrm{~s}$, boundary 1 is more probable because, first, the $0.25-\mathrm{Hz}$ mode is much stronger, and second, two lines on interface $0-1$ have been opened after the 2 nd contingency. $S R I_{1}$ and $S R I_{2}$ for the two boundaries are calculated for $t=0 \sim 85 \mathrm{~s}$ and shown in Fig. 15, which confirms the above conjecture. For boundary $1, S R I_{1}$ exceeds $85 \%$ soon after the 2 nd contingency, exceeds $90 \%$ at $t=80.5 \mathrm{~s}$, and hits $100 \%$ at $t=81.0 \mathrm{~s}$; for boundary $2, S R I_{2}=90 \%$ at $t=83.9 \mathrm{~s}$ and $100 \%$ at $t=84.2 \mathrm{~s}$. At different separation times, separate the system on boundary 1 and shed $3 \mathrm{GW}$ of load in the south island (strategy 11 in 


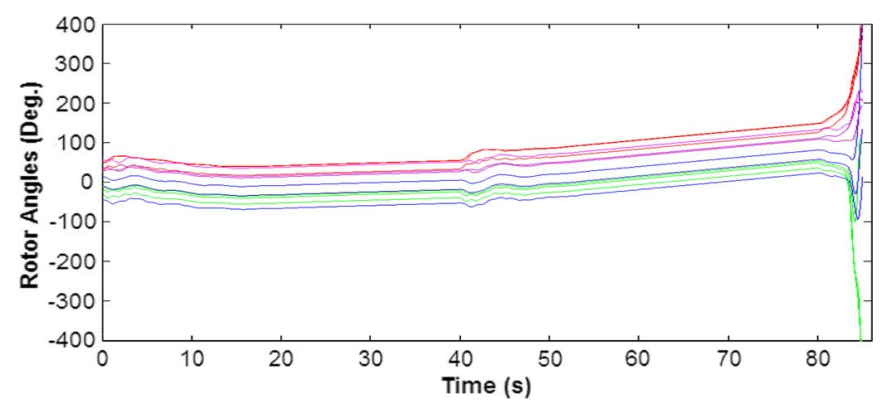

Fig. 12. Rotor angles monitored by 12 synchrophasors.

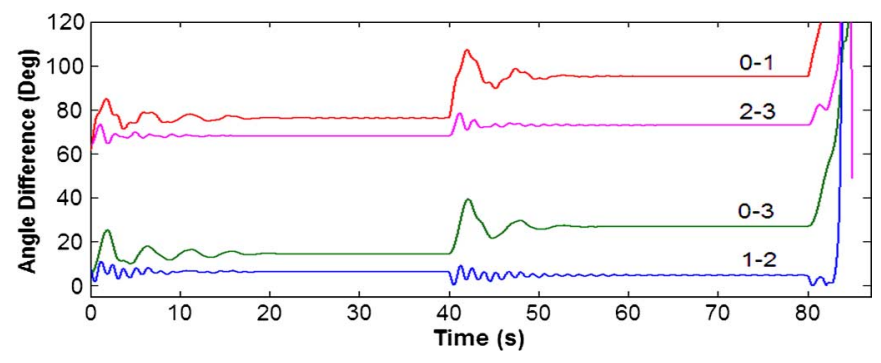

Fig. 13. Angle differences $\delta_{01}, \delta_{03}, \delta_{12}$, and $\delta_{23}$.

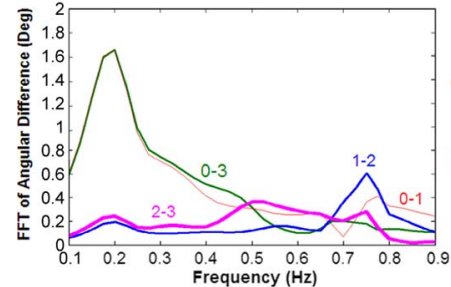

(a)

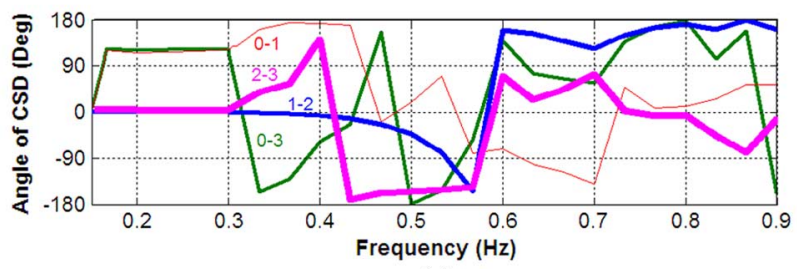

(c)

Fig. 14. Modal analysis results at $t=80$ s. (a) FFT results. (b) $\gamma_{i j}^{2}$. (c) $\beta_{i j}$.

Table IV). Simulation results show that if separation is no later than $t=82.6 \mathrm{~s}$, two stable islands can be formed. Otherwise, similar to Table V, some generators in the south island need to be tripped. Fig. 16 shows the simulation result on separating the system at $t=82.6 \mathrm{~s}$.

\section{E. Separation versus Nonseparation}

In the RTC stage, once the estimated $S R I$ for a boundary is close to $100 \%$ or exceeds a threshold preset for separation, it means that angle separation will happen between two sides of that boundary if no control is added at this time. However, it should be noted that not all out-of-step conditions have to be dealt with by controlled separation unless, first, unintentional separation at the boundary cannot be avoided due to, e.g., cascading outages, or second, analytical or simulation studies have demonstrated that any nonseparation control strategy (e.g.,

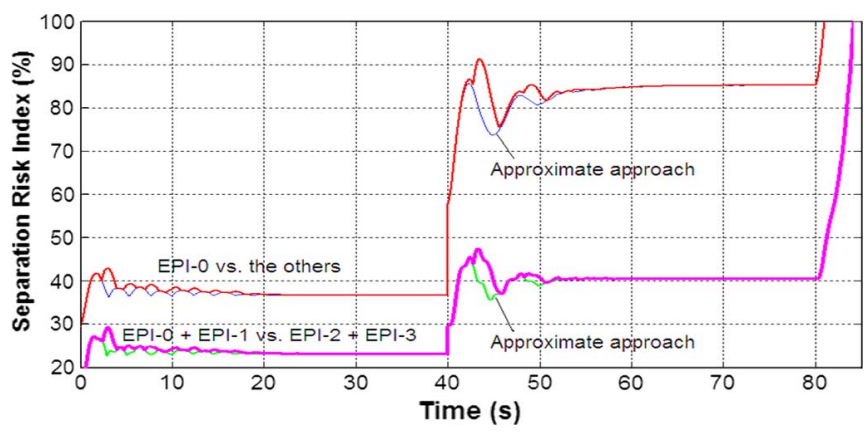

Fig. 15. Separation risk indices for two potential separation boundaries.

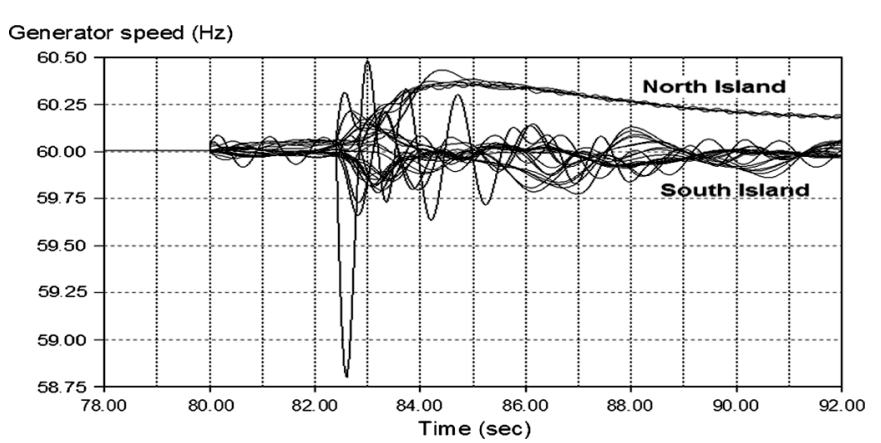

Fig. 16. Generator frequencies after separation at $82.6 \mathrm{~s}$.

reconfiguring load or generation to relieve the stress on that boundary) will lead to more loss. In practice, when out-of-step is predicted, whether or not to separate the system should be studied on a case-by-case basis, and consider costs, effects and even nontechnical factors, which is not this paper's focus.

Nevertheless, the proposed scheme provides a basis not only for studying separation strategies but also for developing a situational awareness system to early indicate where and when out-of-step conditions might occur, such that timely control actions (either separating the system or not) can be taken. The system status after control can continue to be monitored by the techniques in the ONM and RTC stages to check the effects of control. Moreover, those techniques together provide a methodology for offline comparing and evaluating different control strategies facing a specific out-of-step scenario.

For example, as shown in Fig. 10, when the SRI becomes $100 \%$ at $t=201.6 \mathrm{~s}$, separating the system on the boundary with interfaces $0-1$ and $0-3$ and performing Strategy 11 in Table IV can form two stable islands. An alternative strategy is to only perform Strategy 11 without separating the system. Assume that both strategies are performed at $t=201.6 \mathrm{~s}$ and compare them as follows. Simulations show that only performing Strategy 11 can also stabilize the system and lower the $S R I$ on that boundary to around $20 \%$ (as shown in Fig. 17) because the stress on interface $0-1$ is reduced. For two strategies, Figs. 18 and 19, respectively, give the modal analysis results for an immediate 40-s time window after control. From Fig. 18, the system, if not separated, has three potential separation boundaries:

- $0.25 \mathrm{~Hz}$ : interfaces $0-1$ and $0-3$ (EPI-0 versus the others);

- $0.5 \mathrm{~Hz}$ : interfaces 0-3 and 2-3 (EPI-3 versus the others);

- $0.75 \mathrm{~Hz}$ : interfaces $0-1$ and 1-2 (EPI-1 versus the others). 


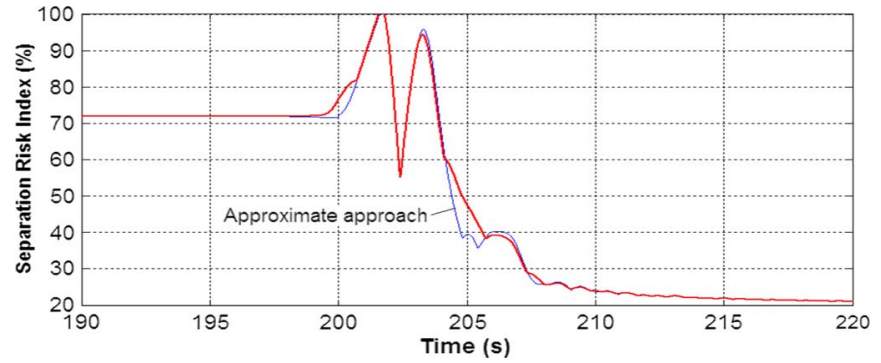

Fig. 17. Separation risk index after only Strategy 11 is performed.

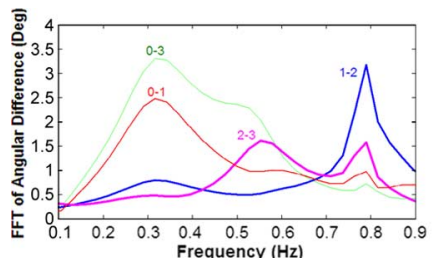

(a)

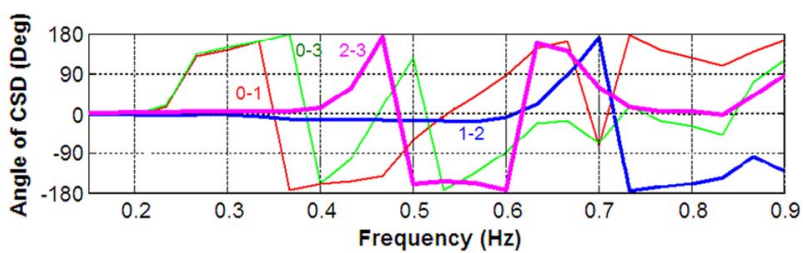

(c)

Fig. 18. Modal analysis results after only performing Strategy 11. (a) FFT results. (b) $\gamma_{i j}^{2}$. (c) $\beta_{i j}$.

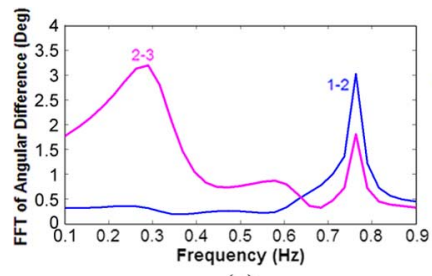

(a)

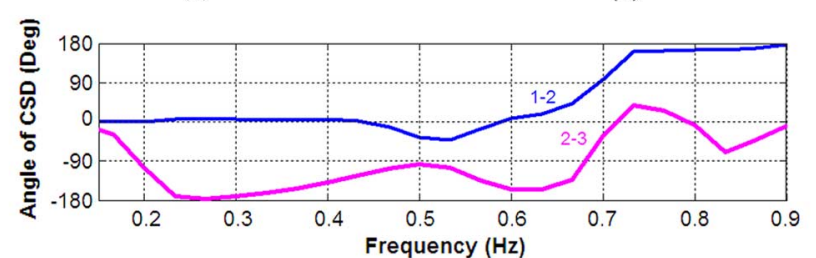

(c)

Fig. 19. Modal analysis results after separation and Strategy 11 is performed. (a) FFT results. (b) $\gamma_{i j}^{2}$. (c) $\beta_{i j}$.

From Fig. 19, after separation, the system's south island (EPI-1+ EPI-2+EPI-3) has two potential separation boundaries:

- $0.25 \mathrm{~Hz}$ : interface 2-3 (EPI-3 versus EPI-1+EPI-2);

- $0.75 \mathrm{~Hz}$ : interface 1-2 (EPI-1 versus EPI-2+EPI-3).

For either strategy, the boundaries have low $S R I$ s after control, and they can continue to be monitored by the scheme in case additional disturbances increase the $S R I$ s.

\section{CONCLUSIONS}

This paper proposed a new unified scheme for controlled separation using synchrophasors. The scheme addresses three critical problems, i.e., "where", "when" and "what", which are usually coupled but are rarely studied together in previous literatures. This scheme adopts a time-staged approach to strategically decoupling the three problems by addressing their sub-problems in different time frames. This scheme also offers a package of techniques for optimizing and predicting potential separation boundaries and estimating risks of angle separation. The algorithms of the scheme may help either perform controlled separation or predict and mitigate unintentional separation. Case studies on the 179-bus system have been performed to demonstrate its performance. Several sensitivity studies on changes in the operating condition, the length of the time window for modal analysis, and separation time are conducted to show the potential of the proposed scheme in online implementation. This paper has been focused on presenting a comprehensive framework for studying the issues related to separation of a power system. If controlled separation is adopted as a defensive strategy against cascading events or severe out-of-step conditions, the planning engineers of the system might utilize the proposed scheme as a basis for investigating separation strategies. The scheme also provides a foundation for developing an early warning and decision support system against severe out-of-step conditions.

\section{ACKNOWLEDGMENT}

The authors would like to thank Dr. Trudnowski and Dr. Hauer for kindly providing MATLAB-based functions on modal analysis. The authors also would like to thank all reviewers for their precious suggestions on improving the paper.

\section{REFERENCES}

[1] 1967, Prevention of Power Failures, U.S. Federal Power Commission, Government Printing Office. Washington, DC. [Online]. Available: http://chnm.gmu.edu/blackout/archive/a_1965.html.

[2] 1978, The Con Edison Power Failure of July 13 and 14, 1977, U.S. Department of Energy. Federal Energy Regulatory Commission. Government Printing Office. Washington, DC. [Online]. Available: http:// chnm.gmu.edu/blackout/archive/a_1977.html.

[3] D. N. Kosterev, C. W. Taylor, and W. A. Mittelstadt, "Model validation for the August 10, 1996 WSCC system outage," IEEE Trans. Power Syst., vol. 14, no. 3, pp. 967-979, Aug. 1999.

[4] U.S.-Canada Power System Outage Task Force, Final Report on the August 14th Blackout in the United States and Canada. [Online]. Available: https://reports.energy.gov.

[5] K. Sun, D. Zheng, and Q. Lu, "Splitting strategies for islanding operation of large-scale power systems using OBDD-based methods," IEEE Trans. Power Syst., vol. 18, no. 2, pp. 912-923, May 2003.

[6] Q. Zhao, K. Sun, and D. Zheng et al., "A study of system splitting strategies for island operation of power system: A two-phase method based on OBDDs," IEEE Trans. Power Syst., vol. 18, no. 4, pp. 1556-1565, Nov. 2003.

[7] K. Sun, D. Zheng, and Q. Lu, "Searching for feasible splitting strategies of controlled system islanding," Proc. Inst. Elect. Eng., Gen., Transm., Distrib., vol. 153, no. 1, pp. 89-98, Jan. 2006.

[8] K. Sun, D. Zheng, and Q. Lu, "A simulation study of OBDD-based proper splitting strategies for power systems under consideration of transient stability," IEEE Trans. Power Syst., vol. 20, no. 1, pp. 389-399, Feb. 2005

[9] X. Li and Q. Zhao, "Parallel implementation of OBDD-based splitting surface search for power system," IEEE Trans. Power Syst., vol. 22, no. 4, pp. 1558-1593, Nov. 2007. 
[10] M. Jin, T. S. Sidhu, and K. Sun, "A new system splitting scheme based on the unified stability control framework," IEEE Trans. Power Syst., vol. 22, no. 1, pp. 433-441, Feb. 2007.

[11] Y. Xue, T. Van Custem, and M. Ribbens-Pavella, "A simple direct method for fast transient stability assessment of large power systems," IEEE Trans. Power Syst., vol. 3, no. 2, pp. 400-412, May 1988.

[12] M. Pavella, D. Ernst, and D. Ruiz-Vega, Transient Stability of Power Systems: A Unified Approach to Assessment and Control. Norwell, MA: Kluwer, 2000.

[13] H. You, V. Vittal, and X. Wang, "Slow coherency-based islanding," IEEE Trans. Power Syst., vol. 19, no. 1, pp. 483-491, Feb. 2004.

[14] X. Wang and V. Vittal, "System islanding using minimal cutsets with minimum net flow," in Proc. IEEE PES Power Systems Conf. Expo., Oct. 10-13, 2004.

[15] B. Yang and V. Vittal, "Slow-coherency-based controlled islanding-A demonstration of the approach on the August 14, 2003 blackout scenario," IEEE Trans. Power Syst., vol. 21, no. 4, pp. 1840-1847, Nov. 2006.

[16] B. Yang and V. Vittal et al., "A novel slow coherency based graph theoretic islanding strategy," in Proc. IEEE PES General Meeting, Jun. 24-28, 2007.

[17] W. Liu, L. Liu, and D. A. Cartes, "Binary particle swarm optimization based defensive islanding of large scale power systems," Int. J. Comput. Sci. Appl., vol. 4, pp. 69-83, 2007.

[18] W. Liu, L. Liu, and D. A. Cartes, "Angle modulated particle swarm optimization based defensive islanding of large scale power systems," in Proc. IEEE PES Power Africa 2007 Conf. Expo., Johannesburg, South Africa, Jul. 16-20, 2007.

[19] R. Diao, V. Vittal, and K. Sun et al., "Decision tree assisted controlled islanding for preventing cascading events," in Proc. IEEE PES Power Systems Conf. Expo. 2009, Seattle, WA.

[20] N. Senroy, G. T. Heydt, and V. Vittal, "Decision tree assisted controlled islanding," IEEE Trans. Power Syst., vol. 21, no. 4, pp. 1790-1797, Nov. 2006.

[21] J. Bertsch, C. Carnal, D. Karlsson, J. Mcdaniel, and K. Vu, "Wide-area protection and power system utilization," Proc. IEEE, vol. 93, no. 5, pp. 997-1003, May 2005.

[22] J. H. Chow, R. Galarza, and P. Accari et al., "Inertial and slow coherency aggregation algorithms for power system dynamic model reduction," IEEE Trans. Power Syst., vol. 10, no. 2, pp. 680-685, May 1995.
[23] D. J. Trudnowski, "Estimating electromechanical mode shape from synchrophasor measurements," IEEE Trans. Power Syst., vol. 23, no. 3, pp. 1188-1195, Aug. 2008.

[24] P. Kundur, Power System Stability and Control. New York: McGrawHill, 1994.

[25] J. F. Hauer, "Application of prony analysis to the determination of modal content and equivalent models for measured power system response," IEEE Trans. Power Syst., vol. 6, no. 3, pp. 1062-1068, Aug. 1991.

[26] K. Hur and S. Santoso, "Estimation of system damping parameters using analytic wavelet transforms," IEEE Trans. Power Del., vol. 24, no. 3, pp. 1302-1309, Jul. 2009.

Kai Sun (M'06) received the B.S. degree in automation and the Ph.D. degree in control science and engineering from Tsinghua University, Beijing, China, in 1999 and 2004, respectively.

He was a postdoctoral research associate at Arizona State University, Tempe, from 2005 to 2007. He is currently a project manager at EPRI, Palo Alto, CA. His research interests include power system stability, dynamics and control, and complex networked system analysis and optimization.

Kyeon Hur (S'04-M'07) received the B.S. and M.S. degrees in electrical engineering from Yonsei University, Seoul, Korea, in 1996 and 1998, respectively, and the Ph.D. degree in electrical and computer engineering from The University of Texas at Austin in 2007.

He was with Samsung Electronics as an R\&D engineer between 1998 and 2003, ERCOT and EPRI between 2007 and 2010. He has rejoined Yonsei University and leads a research group for Smart Grid.

Pei Zhang (M'00-SM'05) received the Ph.D. degree from Imperial College of Science, Technology and Medicine, University of London, London, U.K.

$\mathrm{He}$ is the Program Manager responsible for Grid Operation and Planning area at EPRI, Palo Alto, CA. His current research interests include power system stability and control, reliability and security assessment, and wide-area special protection schemes. 